\title{
Renate Tobies: Felix Klein - Visionen für Mathematik, Anwendungen und Unterricht
}

\author{
Springer Spektrum 2019, XVI + 574 Seiten, ISBN \\ 978-3-662-58748-5, €54,99; eBook ISBN: 978-3-662-58749-2, \\ $€ 42,99$
}

\section{Klaus Volkert}

Online publiziert: 7. Februar 2020

(C) Der/die Autor(en) 2020

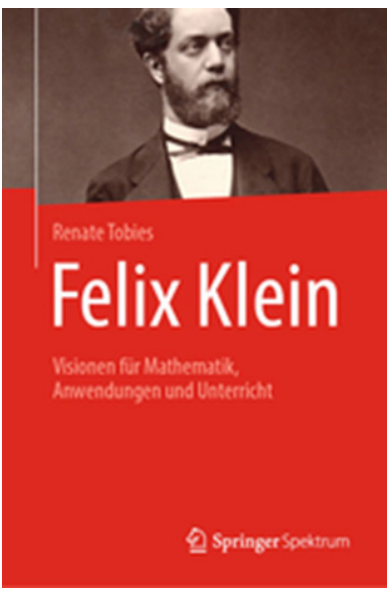

Felix Christian Klein (* Düsseldorf 1849, † Göttingen 1925), der ,große Klein“, war über einen Zeitraum von etwa 50 Jahren ein beherrschender Akteur in der mathematischen Gemeinschaft des deutschsprachigen Raums und darüber hinaus. Politisch gesehen umspannte das Leben des erwachsenen Felix Klein in etwa die Gründung des zweiten deutschen Kaiserreichs bis hin zum Weltkrieg und den anschließenden Anfängen der Weimarer Republik - also einen Zeitraum mit recht konstanten politischen Bedingungen, die erst durch die Revolution von 1918 tiefgreifende Veränderungen erfuhren. Klein war Sohn eines hohen preußischen Beamten, eher konservativ und kaisertreu - aber keineswegs stur, vielmehr anpassungsfähig und vermittelnd, national denkend und doch international agierend.

Bekannt geblieben sind mathematische Errungenschaften, die von ihm erzielt wurden, allen voran das Erlanger Programm, die Cayley-Klein-Metriken, die Kleinsche Flasche und die Kleinsche Vierergruppe, aber auch seine vielseitigen organisatorischen Bemühungen, die entscheidend mithalfen, Göttingen in Allianz mit David Hilbert zum mathematischen (und etwas später auch physikalischen) Weltzentrum zu machen, sowie seine Bemühungen um den Mathematikunterricht, schlagwortartig als „Kleinsche Reform“ bezeichnet. Auch als Buchautor war Klein erfolg-

K. Volkert (ه)

Universität Wuppertal, Wuppertal, Deutschland

E-Mail: klaus.volkert@math.uni-wuppertal.de 
reich. Hier sind u.a. zu nennen seine „Vorlesungen über das Ikosaeder“ (1884-1993 neu hg. mit Kommentaren von P. Slodowy [3]), seine Geometrielehrbücher und seine „Elementarmathematik vom höheren Standpunkt“ (3 Bände 1908, 1909 und 1928). ${ }^{1}$ Klein war wesentlich beteiligt an Mammutprojekten wie der ,Encyklopädie der mathematischen Wissenschaften mit Einschluß ihrer Anwendungen“" ${ }^{2}$ und der ,Kultur der Gegenwart“ (Abteilung mathematische Wissenschaften); er war Organisator - oft auch Mitbegründer - zahlreicher Vereinigungen, insbesondere der Deutschen Mathematiker-Vereinigung (DMV), der Internationalen Mathematischen Unterrichtskommission (IMUK) und deren deutschen Ableger, des Deutschen Ausschuß für mathematischen und naturwissenschaftlichen Unterricht (DAMNU) ${ }^{3}$. Eine detaillierte, umfangreiche Biographie von Klein verspricht somit Aufschlüsse verschiedenster Art.

Klein hat - für Mathematiker eher ungewöhnlich - viel an seiner eigenen Präsentation gearbeitet. Neben kommentierten Wiederveröffentlichungen älterer Texte, bekanntestes Beispiel: das schon genannte Erlanger Programm, vorgelegt von seinem Verfasser anlässlich seines Eintritts in die Philosophische Fakultät der Universität Erlangen im Herbst 1872 und wieder abgedruckt in den „Mathematischen Annalen“ (Band 43, 1893), über seine ,Vorlesungen über die Entwicklung der Mathematik im 19. Jahrhundert" (gehalten während des Ersten Weltkriegs, publiziert erst nach Kleins Tod in zwei Bänden [1926 und 1927]) ${ }^{4}$ bis hin zu seinen „Gesammelten Abhandlungen“ (in drei Bänden, erschienen [1921, 1922, 1923]), welche ausführliche Darlegungen von Klein zu seinen mathematischen Arbeiten, zu deren Entstehung und Zeitumständen enthalten. Diese gut zugänglichen und eingängigen Quellen haben das in der Literatur gängige Klein-Bild zweifellos beeinflusst. Ein Kriterium für eine Biographie wie die von Renate Tobies vorgelegte ist folglich, inwieweit es gelingt, das Bild von Klein durch Aufarbeitung unbekannter oder wenig beachteter Quellen zu vervollständigen und sich von seiner Selbststilisierung frei zu machen. Natürlich gehen auch Gefahren aus von den zahllosen Würdigungen, Ehrungen inklusive Orden, Elogen und Nachrufen, die dem einflussreichen Klein, einem Großen der Wissenschaftswelt seiner Zeit, gewidmet wurden. Bekanntlich wird der größte Querulant bei solchen Anlässen als kooperationsfreudiger Kollege dargestellt - oder man begnügt sich mit netten Allgemeinplätzen. Vorsicht ist folglich angebracht.

Das Buch von Tobies gliedert sich in die Kapitel Einleitung, prägende Gruppen, Professor an der Universität Erlangen, Professor am Polytechnikum München, Professor für Geometrie in Leipzig, Start als Professor in Göttingen 1886-1892, Weichenstellungen 1892/3-1895, Früchte der Bestrebungen 1895-1913, erster Weltkrieg und Nachkriegszeit sowie Schlussbetrachtungen. Ein umfangreicher Anhang mit in-

\footnotetext{
12016 ff wurde eine englische Übersetzung von M. Mengini und G. Schubring publiziert. Vorgängerversionen mancher Bücher von Klein bestanden in autographierten Ausarbeitungen seiner Vorlesungen. Klein, wie auch andere Mathematiker (etwa W. Fiedler in Zürich) nutzte Klein dazu den Steindruck (Lithographie, vgl. p. 154).

2 Dieses Unternehmen stand unter dem ,,wissenschaftliches Obermanagment“ Kleins (S. 356). Kurzangaben beziehen sich hier und im Folgenden immer auf das Buch von R. Tobies [14].

3 Hilfreich ist hier das Abkürzungsverzeichnis in Tobies' Buch S. 553-554 [14].

4 Vgl. auch [11].
} 
teressanten Dokumenten sowie Verzeichnisse runden das Werk ab. Die Darstellung folgt also der Chronologie von Kleins Leben, gegliedert durch seine Berufungen an die Universität Erlangen (1872), an das Polytechnikum München (1875) und die Universitäten Leipzig (1880) sowie Göttingen (1886). Das Jahr 1892 markiert einen wesentliche Einschnitt in der Geschichte der Mathematiklehrstühle im deutschsprachigen Raum - eingeleitet durch die Neubesetzung der Berliner Lehrstühle von Leopold Kronecker und Karl Weierstraß, seinerzeit wohl die prominentesten in Deutschland überhaupt - und die Vakanz in Breslau, entstanden durch den Tod von Heinrich Schröter, einem der wenigen Geometer mit Lehrstuhl in der damaligen universitären Szene.

1895 begann mit der Berufung Hilberts die Geschichte des Erfolgsmodells Göttingen ${ }^{5}$, das in seiner Integration von Mathematik, Physik und Technik, mit seiner Internationalität und seiner Forschungsintensität verbunden mit neuen Formen der Zusammenarbeit eine seinerzeit einmalige Erscheinung war. Klein spielte hier die Rolle des Organisators, des Machers, wie man heute sagen würde, der z.B. schon früh versuchte, Drittmittel von der Industrie zu erhalten - eine der bekanntesten Unternehmungen dieser Art war die „Göttinger Vereinigung zur Förderung der angewandten Physik und Mathematik“, gegründet $1898^{6}$. Wichtigster Bundesgenosse Kleins wurde der Industrielle Henry Theodore Böttinger (1848-1920), leitendes Mitglied der Farbenfabriken vormals Friedr. Bayer und Co. aus Elberfeld, der ähnlich wie Klein breite organisatorische Aktivitäten unterhielt. Böttingers Rolle für Klein und seine Projekte finden ihr Pendant in derjenigen von Friedrich Althoff (1839-1908), Ministerialdirektor und heimlicher preußischer Kultusminister, den Klein schon aus gemeinsamen Tagen als Sanitäter im Deutsch-Französischen Krieg (1870) kannte ${ }^{7}$. Althoff war ein meist treuer Verbündeter Kleins in dessen universitären und schulischen Projekten - eine Ausnahme bildete die Berufung Webers nach Göttingen. Kleins Versuch, Adolf Hurwitz oder David Hilbert nach Göttingen zu holen, scheiterte noch 1892, stattdessen musste er sich mit Heinrich Weber arrangieren ${ }^{8}$. Erst nach dessen Weggang nach Straßburg gelang die Berufung Hilberts, mittlerweile zum Ordinarius aufgerückt in seiner Heimatstadt Königsberg.

Ein interessanter Aspekt von Kleins Wirken als Organisator war seine frühe Wertschätzung von Technik und die daraus folgenden Bemühungen, z. B. um die uneingeschränkte Anerkennung des realistischen Bildungswesens und um die Integration der technischen Hochschule Hannover in die Universität Göttingen ${ }^{9}$. Hier zeigte sich Klein als Denker, der die Zeichen dessen, was man damals „Moderne“ oder auch „Fortschritt“ genannt hätte, wohl verstanden hatte - im Unterschied zu vielen Kollegen, die beim traditionellen Bild von Mathematik als reiner Wissenschaft ver-

\footnotetext{
5 Vgl. hierzu auch [9] part III und part V.

6 Vgl. 8.1.1.

7 Vgl. 2.7.1.

8 Vgl. Abschnitt 6.5 sowie [9], 171-182, der diese Geschichte aus nicht-Kleinscher Perspektive erzählt.

9 Vgl. 6.4.2.
} 
harrten. Ähnliches gilt für Klein als Förderer des Frauenstudiums ${ }^{10}$ und für seine Bemühungen um Internationalität - Reisen und Auslandsaufenthalte sowie ausländische Studierende und Doktoranden inbegriffen, was Klein den Ehrentitel eines Außenministers der deutschen Mathematik einbrachte. ${ }^{11}$ Die organisatorischen, bildungs- und universitätspolitischen Tätigkeiten Kleins werden in Tobies’ Biographie sehr ausführlich dargestellt, viel neues Material aus Briefen und Dokumenten aller Art wird hierzu herangezogen.

Wenden wir uns nun der Mathematik zu. Klein begann bekanntlich seine Karriere als physikalischer Assistent von Julius Plücker in Bonn. Nach dessen Tod und erfolgter Promotion (notgedrungen bei R. Lipschitz, dem verbleibenden Bonner Mathematiker) wandte er sich nach Göttingen, um sich Alfred Clebsch anzuschließen und Mitglied von dessen ,Denkgemeinschaft“ zu werden. ${ }^{12}$ Klein wurde zum Bearbeiter und Herausgeber von Plückers unvollendetem Werk über Liniengeometrie und begann eine wahre Flut von Veröffentlichungen der Öffentlichkeit zu übergeben. ${ }^{13}$ Dabei konnte er die „Mathematischen Annalen“ nutzen, welche Clebsch zusammen mit Carl Neumann 1868 gegründet hatte und die seine Arbeiten unter Clebschs Auspizien offensichtlich freudig veröffentlichen. Diese Zeitschrift sollte später unter Kleins Herausgeberschaft ein wichtiges Instrument für dessen wissenschaftspolitischen Ambitionen, etwa was Internationalität anging, werden. ${ }^{14}$ Kleins Mathematik aus dieser Schaffensperiode ist heute weitgehend vergessen; sie wieder zu erschließen ist eine sehr anspruchsvolle Aufgabe. Verständlicherweise kann eine Biographie hierzu nicht allzu viel beitragen. Das liegt im vorliegenden Fall zum einen auch an der Kürze der Ausführungen, zum andern daran, dass oft die Sprache der damaligen Zeit paraphrasierend verwendet wird, ohne zu erläutern, wie wir den fraglichen Sachverhalt denn heute formulieren würden. ${ }^{15}$ Da helfen auch Elogen ${ }^{16}$ wie die von A. Voss wenig weiter.

Ähnliches gilt für einige andere Abschnitte, in denen auf Kleins Mathematik eingegangen wird. Bei der Diskussion des Erlanger Programmes und der Kleinschen Arbeiten zur nichteuklidischen Geometrie $(1871,1873)^{17}$ wird der aktuelle Stand der Diskussion nur bedingt widergespiegelt. Weder wird klar, dass der Erfolg des Erlan-

\footnotetext{
10 Das Thema ist ubiquitär in Tobies' Buch [14] (u. a. aufgrund der Studentinnen in Kleins Umfeld, die bekannteste hierunter war wohl Grace Chisholm-Young, über die wir einige interessante Dinge erfahren); eine Zusammenfassung bietet 7.6. Schade ist, dass die Leserin und der Leser nicht mehr über Kleins Tochter Elisabeth, verheiratete Staiger, erfährt, die Mathematiklehrerin wurde.

11 Vgl. 8.4.

12 Vgl. 2.4. Clebsch pflegte einen kommunikativen Arbeitsstil und versammelte eine Schar von Schülern/ Mitarbeitern um sich; vgl. S. 40-44. „Denkgemeinschaft“ ist vermutlich an L. Flecks Terminus „Denkkollektiv“ angelehnt, den die Verfasserin schon früh in ihrem Buch zitiert (S. 7).

13 Vgl. 2.4.3.

14 Klein trat 1873 in die Redaktion der Zeitschrift ein, 1876 avancierte er zusammen mit A. Mayer (Leipzig) zum Herausgeber. Vgl. 2.4. Zur Geschichte der „mathematischen Annalen“ vgl. man auch [9], pp. 38-46.

15 Ein Beispiel dafür, wie man so etwas machen kann, liefert die Neuausgabe von Kleins Ikosaederbuch durch P. Slodowy (1993).

16 Vgl. S. 49.

17 Vgl. 2.5.3, 3.1.1 und 6.3.6.
} 
ger Programms doch mehr ein Mythos - nicht zuletzt von Klein selbst gefördert - als eine historische Tatsache ist ${ }^{18}$; die Besprechung von Kleins projektivem „Modell“ wiederholt unhinterfragt die These, dass dieses mit Fragen der Widerspruchsfreiheit befasst gewesen sei. Eine gewisse Einseitigkeit ist in der Darstellung der Kontroverse Klein - Poincaré in Sachen Fuchs'sche Funktionen, also modern gesprochen: automorphe Funktionen, zu verzeichnen: Hier der großherzige Klein, der seine Karten auf den Tisch legt, da der verschwiegene Poincaré, der sich nicht in dieselben schauen lässt. Man kann diese Auseinandersetzung eigentlich nur würdigen, wenn man den mathematischen Kern identifiziert, also Poincarés Entdeckung der Rolle der hyperbolischen Geometrie inklusive derjenigen der Gruppen in diesen Fragen also Ideen, die Klein sicher als seine ureigene Domäne sah. ${ }^{19}$ Auch Kleins Auseinandersetzungen mit Lazarus Fuchs ${ }^{20}$ und Hermann Amandus Schwarz ${ }^{21}$ werden mit einer gewissen Tendenz pro Klein vorgetragen.

Interessant und wichtig ist, dass sich in Kleins frühen Arbeiten zur Linien- und projektiven Geometrie sowie zur Flächentheorie schon ein Bezug zu materialen Modellen ergab, ein Thema, das in Tobies' Biographie zu Recht immer wieder zur Sprache kommt und zu dem sie einige neue Aspekte beizutragen hat. Diese Modelle waren in der Mehrzahl Forschungsobjekte, heißt, sie wurden konstruiert, um abstrakt definierten Objekten, etwa durch Gleichungen gegebene Flächen, Anschaulichkeit zu verleihen. ${ }^{22} 1881$ halfen die Modelle, jetzt allerdings eher didaktische, Klein sogar, seinen ersten (universitären) Assistenten zu bekommen: Walter Dyck in Leipzig. ${ }^{23}$ Solche Informationen machen das vorliegende Buch zu einer wertvollen Quelle für alle, die über die Mathematikgeschichte jener Zeit arbeiten.

An manchen Stellen von Tobies' Biographie finden sich für uns befremdliche Formulierungen, die aber vielleicht bei Klein und seinen Zeitgenossen keinen Anstoß erregt hätten. Hier einige Beispiele: „Mittag-Leffler betrieb eine hinterhältige Politik des gegenseitigen Ausspielens von Mathematikern, ...“ (S. 47), „So wählte ihn [Fr. Schur, K. V.] Klein als Assistenten, nachdem Dycks Weggang feststand, noch bevor Study dreist um die Stelle betteln sollte.“ (S. 208), „... war Study eine egozentrische Natur, die gefördert werden wollte, ohne zu geben." (S. 214) ${ }^{24}$,

\footnotetext{
18 Vgl. etwa [2]; zur Mythenbildung um Kleins Antrittsvorlesung in Erlangen [6].

19 Vgl. die ausführliche Diskussion des Briefwechsels bei Rowe [9], 111-133. Dort wird insbesondere auch Kleins Umgang mit Poincarés Briefen beleuchtet, der nicht eben großzügig war. Vermutlich wird die vom Archiv Henri Poincaré (Nancy) geplante Edition des Briefwechsels von Poincaré mit Mathematikern hier noch mehr Informationen liefern.
}

${ }^{20} \mathrm{Vgl}$. 5.5.5.

${ }^{21} \mathrm{Vgl.} \mathrm{6.2.1.}$

${ }^{22} \mathrm{Vgl}$. dazu [7] und [8] (Modelle als Forschungsobjekte) und [16] (didaktische Verwendung von Modellen, Bezüge zur polytechnischen Tradition). Klein selbst hat anscheinend nie Modelle alleine gebaut, sondern meist bauen lassen - beginnend mit Freund Albert Wenker (vgl. S. 19).

${ }^{23}$ Vgl. p. 194. In München am Polytechnikum gab es, wie damals an Polytechnika üblich, Assistenten. Nach Dycks Weggang von Leipzig nach München übernahm Friedrich Schur die Assistentenstelle bei Klein. Wie ungewöhnlich Kleins Erfolg war, zeigt die Tatsache, dass Hilbert erst nach 1900 seinen ersten bezahlten Assistenten bekam, den er zudem noch mit H. Minkowski teilen musste.

${ }^{24}$ Neben Study war H. A. Schwarz ein Anathema für Klein, vgl. etwa S. 207, wo diesem ein ,unverträglicher Charakter“ bescheinigt wird oder p. 209, wo Schwarzens „,komplizierter“ Charakter zur Sprache kommt. 
„Wenn er [Minkowski, K. V.] auch etwas überheblich auf die Kreiseltheorie blickte, ...“ (S. 393). Einher geht dies mit Wendungen wie „als Plückers Assistent dienen“ $\left(\right.$ S. 36) ${ }^{25}$, ,Klein hatte Sommerfeld zuvor erprobt, ...“ (S. 342), „Für Letzteres konnte Sommerfeld seine Ehefrau Johanna ... einspannen“ (S. 344), „Im einleitenden Band betonte der als Vorsitzender ... in die Spur gesetzte W. Dyck ..." (S. 353), „Er [Klein, K. V.] hatte im März 1886 Sophus Lie dorthin geschickt, ...“ (S. 295). Es entsteht so leicht der Eindruck, als seien Personen Figuren auf einem Schachbrett gewesen, die von Klein gezogen wurden - oft auch zu ihrem eigenen Vorteil: „Als Klein 1875 an das Münchner Polytechnikum wechselte, konnte er Brill auf eine Professur neben sich ziehen." (S. 41). Klein wurde - vor diesem Hintergrund nicht unverständlich - von manchen seiner Weggenossen mit einem gewissen Misstrauen beäugt, wie es sich z. B. in Brills Lebensbeschreibung ${ }^{26}$ und in Wilhelm Fiedlers Briefen zeigt. ${ }^{27}$

Ähnlich wie die mathematischen Gehalte von Kleins Schaffen in dieser Biographie eher undeutlich bleiben, werden die Bemühungen Kleins um eine Unterrichtsreform, die gelegentlich gar als „Kleinsche Unterrichtsreform“ bezeichnet werden, weitgehend auf das Organisatorische reduziert. Die Leserin/der Leser erfährt viel über die diversen Kommissionen, in denen Klein aktiv war ${ }^{28}$; worum es inhaltlich ging, bleibt aber vage. ${ }^{29}$ Hier hätten sich die Meraner Reformvorschläge nebst einer Diskussion, inwieweit diese von Klein beeinflusst waren, angeboten. Klein hat in der Mehrzahl Ansätze aufgegriffen und weiterentwickelt; es scheint aber oft im Buch so, als stammten sie von ihm. ${ }^{30}$ Kleins für ihn wichtiges Eintreten für die Anschauung sowohl in didaktischer als auch in philosophischer und mathematischer Sicht brachte ihm vor einigen Jahren den Titel des „Anti-Modernen“ [4] ein - ähnlich wie z. B. Poincaré und Frege. Interessant in diesem Kontext ist die Einleitung, welche Klein seinen Arbeiten zur ,anschaulichen Geometrie“ im zweiten Band seiner Werke (1922) vorangestellt hat - Fazit und Apologie in ablehnendem Umfeld zugleich.

Die Kämpfe gegen die Zeitläufte, die Kleins Intentionen zuwider liefen, indem sie die Wende zur abstrakten und formalen Mathematik begünstigten, werden in Tobies' Biographie mehrfach angesprochen. ${ }^{31}$ Mit David Hilbert war einer der wichtigsten Vorkämpfer dieser Richtung gar im eigenen Institut. Kleins Eintreten für die angewandte Mathematik ${ }^{32}$, insbesondere für deren Berücksichtigung in der Lehrerbildung und im gymnasialen Unterricht, erscheint im übrigen sehr aktuell. Hervorgehoben seien schließlich Kleins Bemühungen, die Didaktik an den Universitäten, insbeson-

\footnotetext{
${ }^{25}$ Es geht um Plückers Assistent Emil Budde. Vgl. auch S. 434, wo von Schimmack die Rede ist, der „Klein 1903-1905 als Assistent diente“.

${ }^{26}$ Brill wird übrigens S. 184 unterstellt, er habe Klein beneidet. Die unveröffentlichte Lebensbeschreibung Brills befindet sich im Archiv der TU München.

27 Vgl. [1].

${ }^{28}$ Eine nützliche Übersicht zu den diversen Gremien und Kommissionen, in denen Klein Mitglied war, findet sich S. 426.

${ }^{29} \mathrm{Vgl}$. 8.3.4.

30 Beispielsweise heißt es S. 433: „Klein erhob die Mathematikdidaktik zu einer selbständigen Disziplin“ ein Unternehmen, an dem viele beteiligt waren. Klein nutzte vor allem seinen institutionellen Einfluss.

31 Vgl. 8.2.

${ }^{32}$ Zwei Beispiele: Versicherungsmathematik (vgl. 7.7) und Luftfahrtforschung (vgl. 8.1.3).
} 
dere natürlich der Göttinger, als Lehrfach zu etablieren, durch die Habilitation von R. Schimmack (S. 434) und dessen Einbezug in die Lehre. ${ }^{33}$ Ein wichtiger Weggefährte Kleins im Bereich Mathematikunterricht war Walter Lietzmann, der an vielen Stellen des Buches zur Sprache kommt und sicher weitere Forschungen verdiente. Auch Friedrich Drenckhahn und Paul Zühlke, Doktoranden von O. Staude, wurden von Klein zur Didaktik geführt; ersterer wurde einer der Pioniere der Lehre der Mathematikdidaktik an Pädagogischen Hochschulen. ${ }^{34}$

Trotz der Ausführlichkeit dieser Biographie bleibt Kleins Persönlichkeit, sein Leben als Mann und Mensch, recht undeutlich. Persönliche Informationen fehlen weitgehend; die Leserin/der Leser erfährt z. B. fast gar nichts über die Beziehungen zu seiner Ehefrau Anna, geborene Hegel und Enkelin des Philosophen, oder zu seinen Kindern. Freunde werden erwähnt - eventuell mit dem Hinweis, dass diese „Duz-Freunde“ Kleins gewesen seien ${ }^{35}$ - aber wie Klein mit ihnen umgegangen ist, bleibt oft unklar. Tobies' Biographie markiert hier geradezu die Antithese zu Constance Reids Hilbert-Biographie [5], die auch und gerade in solchen Hinsichten - man könnte sie persönlich nennen - sehr viel zu bieten hat. Der Unterschied betrifft aber auch einen weiteren wichtigen Aspekt. Während bekanntlich Reid nur höchst selten Belege und Quellen für ihre Schilderungen liefert, ist bei Tobies alles genauestens belegt - sehr zur Freude der Historikerin/des Historikers.

Ein persönlicher Aspekt kommt allerdings doch und völlig zu Recht ausführlich in der Biographie zur Sprache, nämlich Kleins gesundheitliche Probleme (vor allem Asthma, was seine bevorzugten Aufenthalte auf Nordsee-Inseln erklärt). Tobies zeigt auf, dass sich diese durch Kleins ganzes Leben hindurchziehen und letztlich auch (mit) zu seinem für damalige Verhältnisse frühzeitigen Rückzug aus dem Lehramt führten (31.12.1912). Insbesondere macht sie deutlich, dass Kleins Bericht von seinem gesundheitlichen Zusammenbruch (1882) und dem damit verbundenen Verlust an mathematischer Produktivität (,,soziale Wirksamkeit als Ersatz für das verlorene Genie“" wie Klein notierte $^{36}$ ) vor diesem Hintergrund nicht sehr überzeugend ist. ${ }^{37}$

Klein enthielt sich allzu deutlicher politischen Äußerungen, er trat auch nie einer Partei bei. Als Mitglied des Preußischen Herrenhauses vertrat er die Universität Göttingen. Andererseits bewegte er sich auf politiknahem Parkett und hatte enge Kontakte zu führenden Kreisen der Industrie. Offensichtlich war er geschickt und wusste, sich taktisch klug zu verhalten. Das ist zumindest der Eindruck, den man bei der Lektüre der ersten 450 Seiten von Tobies' Biographie gewinnt. Dann aber wir sind in der Zeit kurz nach Ausbruch des Weltkrieges angelangt - erwartet die Leserin, den Leser eine Überraschung, wenn es um den berüchtigten „Aufruf an die Kulturwelt", das sogenannte Manifest der $93^{38}$, geht:

\footnotetext{
33 Vgl. auch die wenig bekannte Information zur von Klein betriebenen Habilitation von Conrad Müller für „Mathematik, namentlich Geschichte der Mathematik“ 1908 in Göttingen (S. 412).

34 Vgl. S. 435. Zu Drenckhahn vgl. man [13].

35 Etwa Carl Linde und Alfred Stern.

36 Vgl. S. 103.

37 Vgl. S. 200.

38 Der Text des Aufrufs ist beispielsweise in der Wikipedia nachzulesen. Hintergrund war der deutsche Überfall auf Belgien, die dort verübten Taten und die internationale Kritik an diesen.
} 
Wie Klein später seiner Schülerin Grace Chisholm Young ${ }^{39}$ erklärte, war er aber - wie Max Planck u.a. - nur telegraphisch um seine Unterschrift ersucht worden. Klein kannte den Text vor dem Druck nicht. Er hatte (naiv) angenommen, es wäre ein Text, der helfen könne, vorhandene Wogen zu glätten. ${ }^{40}$

Schwer zu glauben; mag dem sein, wie es will, Fakt bleibt, dass Klein der einzige Mathematiker war, der diesen Aufruf unterschrieb. ${ }^{41}$ Bemerkenswert bleibt allerdings, dass Klein anscheinend keinen Versuch unternahm, seine Unterschrift zurückzuziehen oder den Text zu widerrufen.

Für ihr Buch hat die Verfasserin eine große Zahl von Quellen ausgewertet, insbesondere Briefe von und an Klein (Darboux, Hilbert, Hurwitz, Lie, um nur einige zu nennen), daneben handschriftliche Aufzeichnungen Kleins, offizielle Dokumente wie Berichte und vieles mehr. Es bildet gewissermaßen die Summa einer langjährigen und engagierten Beschäftigung mit Klein. ${ }^{42}$ Hätte ich einen Wunsch frei, so würde ich mir noch eine Bibliographie der vielen Werke Kleins wünschen, aber das wäre sicherlich viel - wenn auch nützliche - Arbeit. Bemerkenswert und bislang nicht viel beachtet ist Kleins Verhältnis zu Hurwitz, seinem vielleicht besten Schüler. Während er viele andere Schüler wie Dyck, Fricke und Sommerfeld für seine Zwecke einsetzte - Sommerfeld sprach gar vom „Felix Dienst“43 - nahm er bei Hurwitz hiervon Abstand. Hurwitz spielte dann vor allem die Rolle des fachlichen Beraters, den Klein heranzog, als er versuchte, sich in neue Gebiete im Bereich der Funktionentheorie und der analytischen Zahlentheorie einzuarbeiten. Allerdings hatte Hurwitz wie auch F. Lindemann und H. Minkowski dann das Nachsehen gegenüber Hilbert, als es 1895 um die Nachfolge von Weber in Göttingen ging. ${ }^{44}$ Klein förderte junge Kollegen, aber Klein entschied auch, wann er wen förderte. „Einmal Kleins Favorit, immer Kleins Favorit“" galt keineswegs - was doch wieder an die Spielsteine auf dem Schachbrett denken lässt.

Das Geschilderte stellt nur eine Auswahl aus dieser sehr reichhaltigen, lesenswerten Biographie dar, die der Leserin/dem Leser aber einige Mühe bei der Lektüre abverlangt. Diese Lektüre eröffnet ein leicht pathetisches Zitat von Otto

\footnotetext{
39 Die Engländerin Grace Chisholm (1868-1944), die in Cambridge an einem Frauencollege (Girton College) studiert hatte, war Kleins erste Doktorandin (,Algebraisch-gruppentheoretische Untersuchungen zur sphärischen Trigonometrie“, 1895), mit der er auch weiterhin in Kontakt blieb. Sie heiratete später den Mathematiker William Young und nahm den Doppelnamen Chisholm-Young an. Ihr wohl bekanntestes Buch (zusammen mit ihrem Mann veröffentlicht) „Beginner's book of geometry“ (London, 1905) - neben dem ebenfalls mit ihrem Mann veröffentlichten fachwissenschaftlichen Werk ,The theory of sets of points“ (1906) - geht nach Angabe von Tobies ([14], S. 271) auf eine Anregung von Klein zurück. Dieser sorgte auch für die deutsche Übersetzung als „Der kleine Geometer“ durch das Ehepaar Bernstein (1908). Klein soll den Plan einer englischsprachigen Ausgabe der „Encyklopädie“ mit dem Ehepaar Young diskutiert haben (nach [14], S. 356 n. 85). In beiden Fällen werden allerdings bei Tobies keine Belege genannt.

40 S. 452.

41 Die etwas später veröffentlichte „Erklärung der Hochschullehrer des Deutschen Reiches“ ähnlichen Tenors unterschrieben mehr als 3000 Universitätslehrer, unter ihnen D. Hilbert und G. Landau. Vgl. [15].

42 Ein erster Beleg für diese war Tobies 1981 [14]. Weiterhin vgl. man das Literaturverzeichnis (S. 545-548) von Tobies' Biographie, wo man zahlreiche Artikel dieser Autorin findet.

43 S. 400.

44 Vgl. 7.9.
} 
Blumenthal ${ }^{45}$ aus dessen Rede anlässlich der Einweihung einer Gedenktafel für Felix Klein $(1928)^{46}$

Wer im Gedächtnis der großen Welt leben soll, muss auf die große Welt gewirkt haben.

Ich denke, der „große Klein“ nimmt dies zustimmend zur Kenntnis - und die Biographie von R. Tobies trägt viel dazu bei, dass wir jetzt (noch) mehr von Kleins Schaffen wissen - ein Schaffen, das von einer ungeheuren Bewunderung der Kreativität getragen war, und dem Bewusstsein, sie selbst nicht (mehr) zu besitzen. Tobies hat unser Bild von Klein in vielen Punkten - vor allem, was seine organisatorischen Aktivitäten betrifft - vervollständigt, Spuren von dessen Selbststilisierung und von seiner Stilisierung durch andere sind aber noch nicht gänzlich verschwunden.

Funding Open Access funding provided by Projekt DEAL.

Open Access Dieser Artikel wird unter der Creative Commons Namensnennung 4.0 International Lizenz veröffentlicht, welche die Nutzung, Vervielfältigung, Bearbeitung, Verbreitung und Wiedergabe in jeglichem Medium und Format erlaubt, sofern Sie den/die ursprünglichen Autor(en) und die Quelle ordnungsgemäß nennen, einen Link zur Creative Commons Lizenz beifügen und angeben, ob Änderungen vorgenommen wurden.

Die in diesem Artikel enthaltenen Bilder und sonstiges Drittmaterial unterliegen ebenfalls der genannten Creative Commons Lizenz, sofern sich aus der Abbildungslegende nichts anderes ergibt. Sofern das betreffende Material nicht unter der genannten Creative Commons Lizenz steht und die betreffende Handlung nicht nach gesetzlichen Vorschriften erlaubt ist, ist für die oben aufgeführten Weiterverwendungen des Materials die Einwilligung des jeweiligen Rechteinhabers einzuholen.

Weitere Details zur Lizenz entnehmen Sie bitte der Lizenzinformation auf http://creativecommons.org/ licenses/by/4.0/deed.de.

\section{Literatur}

1. Confalonieri, S./Schmidt, P.-M./Volkert, K.: Der Briefwechsel von Wilhelm Fiedler mit Alfred Clebsch, Felix Klein und italienischen Mathematikern. Siegener Beiträge zur Geschichte und Philosophie der Mathematik, Bd. 12 (2020)

2. Hawkins, Th: The Erlanger Programm of Felix Klein: reflections on its place in the history of mathematics. Hist. Math. 11, 442-470 (1984)

3. Klein, F.: Vorlesungen über das Ikosaeder und die Auflösung der Gleichungen fünften Grades. Birkhäuser, Teubner, Basel, Boston, Berlin, Stuttgart, Leipzig (1993). Herausgegeben mit einer Einleitung und mit Kommentaren versehen von Peter Slodowy

4. Mehrtens, H.: Moderne - Sprache - Mathematik. Suhrkamp, Frankfurt a. M (1990)

5. Reid, C.: Hilbert. Springer, New York (1970)

6. Rowe, D.: Felix Klein's „Erlanger Antrittsrede“. Hist. Math. 12, 123-141 (1985)

7. Rowe, D.: Mathematical models as artefacts for research: Felix Klein and the case of Kummer surfaces. Math Semesterber 60, 1-24 (2013)

8. Rowe, D.E.: On building and interpreting models: four historical case studies. Math. Intell. 39(2), 6-14 (2017)

9. Rowe, D.: A richer picture of mathematics. Springer Nature, Cham (2018)

\footnotetext{
45 Die Sammlungen von Briefen und Schriften Otto Blumenthals ([10] und [12]) bieten übrigens viel lesenswertes Material zu Klein.

46 P. V.
} 
10. Rowe, D.: Otto Blumenthal: Ausgewählte Briefe und Schriften I. 1897-1918. Springer Spektrum, Berlin (2018)

11. Rowe, D.: Relativity in the Making, 1916-1918: Rudolf J. Humm in Göttingen and Berlin. Mathematische Semesterberichte, Bd. 67 (2020)

12. Rowe, D., Felsch, V.: Otto Blumenthal: Ausgewählte Briefe und Schriften II. 1919-1944. Springer Spektrum, Berlin (2019)

13. Schönbeck, J.: Von Rostock nach Flensburg - Friedrich Drenckhahn 1894-1977, Demokratische Geschichte. Jahrbuch für Schleswig-Holstein, Bd. 27, S. 89-120 (2016)

14. Tobies, R.: Felix Klein. Teubner, Leipzig (1981)

15. Tollmien, C.: „Der Krieg der Geister“ in der Provinz - das Beispiel der Universität Göttingen 1914-1919. Göttinger Jahrbuch, Bd. 41., S. 137-209 (1993)

16. Volkert, K.: Mathematische Modelle und die polytechnische Tradition. Siegener Beiträge zur Geschichte und Philosophie der Mathematik, Bd. 10., S. 161-202 (2017) 